\title{
Effects of similar intakes of marine $n-3$ fatty acids from enriched food products and fish oil on cardiovascular risk markers in healthy human subjects
}

\author{
Bente Kirkhus ${ }^{1,2_{*}}$, Amandine Lamglait ${ }^{2}$, Karl-Erik Eilertsen ${ }^{3}$, Eva Falch ${ }^{2}$, Trond Haider ${ }^{4}$, Hogne Vik ${ }^{5}$, \\ Nils Hoem ${ }^{5}$, Tor-Arne Hagve ${ }^{6,7}$, Samar Basu ${ }^{8,9}$, Elisabeth Olsen ${ }^{10}$, Ingebjørg Seljeflot ${ }^{11}$, Lena Nyberg $^{12}$, \\ Elisabeth Elind $^{13}$ and Stine M. Ulven ${ }^{13}$ \\ ${ }^{1}$ Nofima AS, Norwegian Institute of Food, Fisheries and Aquaculture Research, Osloveien 1, $1430 \AA$ As, Norway \\ ${ }^{2}$ Mills DA, Oslo, Norway \\ ${ }^{3}$ Faculty of Biosciences, Fishery and Economics, University of Tromsø, Tromsø, Norway \\ ${ }^{4}$ Link Medical Research AS, Oslo, Norway \\ ${ }^{5}$ Aker BioMarine Antarctic AS, Oslo, Norway \\ ${ }^{6}$ Department of Medical Biochemistry, Oslo University Hospital, Rikshospitalet, Oslo, Norway \\ ${ }^{7}$ Center of Laboratory Medicine, Akershus University Hospital, Lørenskog, Norway \\ ${ }^{8}$ Department of Public Health and Caring Sciences, Uppsala University, Uppsala SE-755 85, Sweden \\ ${ }^{9}$ Laboratoire de Biochimie, Biologie Moléculaire et Nutrition, Faculté de Pharmacy, Université d'Auvergne, \\ 28 Place Henri-Dunant, 63001 Clermont-Ferrand, France \\ ${ }^{10}$ Denomega Nutritional Oils, Sarpsborg, Norway \\ ${ }^{11}$ Department of Cardiology, Oslo University Hospital, Ullevål, Oslo, Norway \\ ${ }^{12}$ Skånemejerier, Malmø, Sweden \\ ${ }^{13}$ Faculty of Health, Nutrition and Management, Akershus University College, Kjeller, Norway \\ (Submitted 19 October 2010 - Final revision received 13 July 2011 - Accepted 13 July 2011 - First published online 15 September 2011)
}

\section{Abstract}

There is convincing evidence that consumption of fish and fish oil rich in long-chain (LC) $n-3$ PUFA ( $n-3$ LCPUFA), EPA (20:5n-3) and DHA $(22: 6 n-3)$ reduce the risk of CHD. The aim of the present study was to investigate whether $n-3$ LCPUFA-enriched food products provide similar beneficial effects as fish oil with regard to incorporation into plasma lipids and effects on cardiovascular risk markers. A parallel 7-week intervention trial was performed where 159 healthy men and women were randomised to consume either $34 \mathrm{~g}$ fish pâté ( $n$ 44), $500 \mathrm{ml}$ fruit juice $(n$ 38) or three capsules of concentrated fish oil $(n 40)$, all contributing to a daily intake of approximately $1 \mathrm{~g}$ EPA and DHA. A fourth group did not receive any supplementation or food product and served as controls ( $n$ 37). Plasma fatty acid composition, serum lipids, and markers of inflammation and oxidative stress were measured. Compared with the control group, plasma $n$ - 3 LCPUFA and EPA:arachidonic acid ratio increased equally in all intervention groups. However, no significant changes in blood lipids and markers of inflammation and oxidative stress were observed. In conclusion, enriched fish pâté and fruit juice represent suitable delivery systems for $n-3$ LCPUFA. However, although the dose given is known to reduce the risk of CVD, no significant changes were observed on cardiovascular risk markers in this healthy population.

Key words: Foods: Fish oil: $\boldsymbol{n}$-3 Fatty acids: Intervention: Serum lipids: Inflammation: Oxidative stress

The health benefits of long-chain (LC) $n$-3 PUFA ( $n$-3 LCPUFA) of marine origin, in particular EPA $(20: 5 n-3)$ and DHA (22:6n-3), are well documented, indicating protective effects on CVD, autoimmune and mental disorders ${ }^{(1-5)} \cdot n-3$ LCPUFA appear to reduce the risk of CVD through a wide range of beneficial effects, including anti-atherothrombogenic effect and reduction in serum TAG, whereas there are some inconsistencies regarding the effects on arrhythmia, hypertension and inflammation ${ }^{(6-17)}$. However, $n$-3 LCPUFA are prone to oxidation, which may lead to increased susceptibility to oxidation and atherogenicity of LDL-cholesterol, and increased risk of $\mathrm{CVD}^{(18-20)}$. High levels of F2-isoprostanes, formed

Abbreviations: AA, arachidonic acid; LC, long chain.

*Corresponding author: Dr B. Kirkhus, fax +47 649703 33, email bente.kirkhus@nofima.no 
from the free radical-induced peroxidation of membranebound arachidonic acid (AA, $20: 4 n-6$ ), have been associated with higher cardiovascular risk $^{(21-24)}$. Still, the role of $n-3$ LCPUFA in oxidative stress is unclear.

In the literature, recommended daily intakes of $n-3$ LCPUFA vary from $200 \mathrm{mg}$ to $1 \mathrm{~g} \mathrm{EPA}$ and $\mathrm{DHA}^{(25)}$. Recent evidence shows that the intake of EPA and DHA is inversely related to cardiovascular risk in a dose-dependent manner up to about $250 \mathrm{mg} / \mathrm{d}$ in healthy populations, and intake of $1 \mathrm{~g} / \mathrm{d}$ is associated with a marked protection from sudden cardiac death $^{(2,6,26,27)}$. A growing market for $n$-3 LCPUFA-fortified foods may provide recommended amounts for people whose diet is inadequate. However, whether consumption of such products provides the same health benefits as fish consumption or fish oil supplementation has to be established. There is an ongoing discussion about the preferred 'delivery system' for $n-3$ fatty acids, i.e. food $v$. supplement, with respect to bioavailability and physiological function. The molecular form of the lipids, the food matrix and possible interactions with other food components may have an impact on the absorption and incorporation of EPA and DHA into plasma lipids. Some studies have indicated that fish consumption is more effective in increasing serum concentrations of $n-3$ LCPUFA than fish oil ${ }^{(28,29)}$, whereas others have shown almost identical increases when comparing equal intakes of EPA and DHA from fish and capsules ${ }^{(30)}$. In a single-meal study, fish oil incorporated into food products was absorbed differently from the same fish oil in capsules, and the rate of absorption varied depending on the food matrix ${ }^{(31)}$. Both level and type of emulsifiers and phospholipids in foods may influence lipid bioavailability ${ }^{(32)}$; still, it is unclear whether steady-state levels of plasma EPA and DHA, obtained after regular daily intake, are also influenced by the food matrix.

In a previous intervention study, intake of fish pâté enriched in EPA and DHA indicated efficient incorporation into blood lipids and favourable effects on inflammatory markers ${ }^{(33)}$. In the present study, the same fish pâté and n-3 LCPUFAenriched fruit juice were compared with fish oil with regard to incorporation into plasma lipids and effects on cardiovascular risk markers, e.g. serum lipids, plasma fatty acid profile, and markers of inflammation and oxidative stress. The aim was to investigate whether $n-3$ LCPUFA-enriched food products provide similar beneficial effects as fish oil when consuming $1 \mathrm{~g} / \mathrm{d}$ EPA and DHA, a recommended intake known to reduce the risk of CVD.

\section{Experimental methods}

\section{Participants}

Participants were recruited by local advertising in the community of Akershus, Norway. Men and women, aged 18-70 years, with normal to slightly increased cholesterol and TAG levels (total cholesterol $<7.5 \mathrm{mmol} / 1$ and TAG $<4.0 \mathrm{mmol} / \mathrm{l}$ ) and $\mathrm{C}$-reactive protein $(<10 \mathrm{mg} / \mathrm{l})$ were included. Exclusion criteria were regular fatty fish consumption $(>1 /$ week of salmon, trout, herring, sardine and mackerel), diagnosis of familial hypercholesterolaemia, secondary hyperlipidaemia, chronic rheumatoid disease, coronary, peripheral or cerebrovascular disease within the previous 3 months of inclusion, BMI $\geq 30 \mathrm{~kg} / \mathrm{m}^{2}$, hypertension ( $\geq 160 / 100 \mathrm{mmHg}$ ), use of medication to lower serum lipids, blood pressure and inflammation, pregnancy and lactation. Subjects who used n-3 LCPUFA supplements regularly were asked to terminate the use of supplements 2 months before inclusion ( $n$ 19). The 2 months washout period was considered appropriate based on the reduced level of EPA and DHA in serum cholesteryl esters reported previously during washout after $n-3$ supplementation $^{(34)}$. The study was conducted according to the guidelines in the Declaration of Helsinki and approved by the regional Ethics Committee. Written informed consent was obtained from all participants. A validated $\mathrm{FFQ}^{(35)}$ was used in order to check the background diet of the subjects. All subjects, including the control group, were instructed by a nutritionist not to change their habitual diet (including changes in fatty fish intake and use of $n$-3 supplements), to keep a stable weight and not to change their lifestyle during the intervention period. The intervention period did not include summer or Christmas holidays.

\section{Study design}

The study was part of a larger study, which has been published previously ${ }^{(36)}$. It was a single-centre intervention study with an open, randomised, parallel-group design conducted at the Akershus University College, Kjeller, Norway. Intervention products to be tested were $n-3$ LCPUFA-enriched fish pâté, $n$-3 LCPUFA-enriched fruit juice and concentrated fish oil in capsules. In total, 369 phone calls were received and 207 subjects were invited to a screening visit (visit 1). Subjects eating fatty fish regularly more than once per week were excluded at the phone interviews. Screening of subjects ( $n$ 199) was performed within 3 weeks before inclusion in order to identify subjects who satisfied the eligibility criteria ( $n$ 179). The evaluation conducted at screening included assessment of C-reactive protein and serum lipids. Demographic information (age, sex and ethnicity), concomitant medication and medical history were registered, and a brief physical examination (weight, height and blood pressure) was conducted. Subjects who satisfied the eligibility criteria ( $n$ 179) were randomised into four study groups. Before the baseline visit (visit 2), nine subjects were lost, whereas eleven subjects dropped out during the study period. In the $n-3$ LCPUFA-enriched fruit juice group, two subjects did not manage to consume the product, in the $n$ - 3 LCPUFA-enriched fish pâté group there were five dropouts (two did not manage to eat the product, two got clinical symptoms such as stomach pain and nausea and one was lost to follow-up) and in the control group four subjects were lost to follow-up. A total of 159 subjects finished the study, and they had consumed either $500 \mathrm{ml} n$-3 LCPUFA-enriched fruit juice ( $n 38$ ), $34 \mathrm{~g}$ $n$-3 LCPUFA-enriched fish pâté ( $n$ 44) or three capsules of fish oil ( $n$ 40) daily for 7 weeks in order to obtain an intake of approximately $1 \mathrm{~g} / \mathrm{d}$ EPA and DHA (Table 1$)$. A control group that did not receive any products ( $n$ 37) was included 
Table 1. Fatty acid composition of the study products (percentage of fatty acids)

\begin{tabular}{|c|c|c|c|c|c|c|}
\hline \multirow[b]{2}{*}{ Fatty acids } & \multicolumn{2}{|c|}{ Fruit juice (500 ml) } & \multicolumn{2}{|c|}{ Fish pâté (34 g) } & \multicolumn{2}{|c|}{ Fish oil (three capsules) } \\
\hline & $\%$ & Daily dose (g) & $\%$ & Daily dose (g) & $\%$ & Daily dose $(\mathrm{g})$ \\
\hline $14: 0$ & $1 \cdot 8$ & 0.09 & 1.9 & 0.18 & 3.2 & 0.05 \\
\hline $16: 0$ & $14 \cdot 0$ & 0.65 & 8.75 & 0.80 & $7 \cdot 8$ & 0.13 \\
\hline $18: 0$ & $4 \cdot 3$ & 2.05 & $3 \cdot 7$ & 0.33 & $2 \cdot 6$ & 0.04 \\
\hline $16: 1 n-7$ & 3.2 & 0.15 & $2 \cdot 8$ & 0.25 & 3.9 & 0.07 \\
\hline $18: 1 n-7$ & 3.4 & 0.16 & 3.75 & 0.34 & $2 \cdot 2$ & 0.04 \\
\hline $18: 1 n-9$ & $26 \cdot 0$ & 1.25 & 36.5 & $3 \cdot 28$ & 6.1 & $0 \cdot 10$ \\
\hline $20: 1 n-9$ & 1.6 & 0.08 & 3.05 & 0.27 & $2 \cdot 0$ & 0.03 \\
\hline $22: 1 n-11$ & 1.4 & 0.07 & 1.9 & 0.17 & 2.5 & 0.04 \\
\hline $18: 2 n-6$ & $19 \cdot 0$ & 0.90 & $12 \cdot 0$ & $1 \cdot 11$ & 0.8 & 0.01 \\
\hline $18: 3 n-3$ & 1.3 & 0.06 & $5 \cdot 1$ & 0.46 & 0.5 & 0.01 \\
\hline $18: 4 n-3$ & 0.9 & 0.05 & 0.75 & 0.07 & 1.9 & 0.03 \\
\hline $20: 4 n-6(\mathrm{AA})$ & 0.6 & 0.03 & 0.25 & 0.04 & 1.5 & 0.03 \\
\hline $20: 5 n-3$ (EPA) & 8.7 & 0.41 & $6 \cdot 65$ & 0.60 & $27 \cdot 0$ & 0.45 \\
\hline $22: 5 n-3$ (DPA) & 1.5 & 0.07 & 1.65 & 0.15 & 4.8 & 0.08 \\
\hline $22: 6 n-3(\mathrm{DHA})$ & 7.6 & 0.35 & 5.95 & 0.54 & 24.0 & 0.41 \\
\hline $\mathrm{EPA}+\mathrm{DHA}$ & $16 \cdot 3$ & 0.8 & $12 \cdot 6$ & $1 \cdot 1$ & $51 \cdot 0$ & 0.9 \\
\hline Total fat & & $5 \cdot 0$ & & $9 \cdot 6$ & & 1.8 \\
\hline
\end{tabular}

AA, arachidonic acid; DPA, docosapentaenoic acid.

to account for seasonal variation. The participants were advised to include fish pâté and fruit juice in meals where they commonly used bread or a beverage and the capsules to be consumed at breakfast or other regular meals. All products were delivered free of charge. At the end of the 7-week intervention period, the subjects met for a final visit (visit 3). Blood and urine samples were collected at visits 2 and 3 for assessments of efficacy. A change in concomitant medication and smoking habits from baseline and clinical symptoms during the intervention period were registered in the case report form at each visit. In total, eighteen subjects were using a stable dose of hormonal contraceptives before and during the study, and eight subjects were using drugs against allergies. The number of subjects taking medication was equally distributed among the study groups. The total number of smokers was eighteen. The number of cigarettes per $\mathrm{d}$ varied among the subjects (from 1 up to 20 per d), but the number was stable for each subject before and during the study. Compliance was ensured through regular telephone contact with the participants and monitored by collecting leftover study products and empty containers at visit 3. Compliance was satisfactory in all groups; $94 \%$ in the fish pâté group, $100 \%$ in the juice group and $100 \%$ in the fish oil group.

\section{Products}

Fish pâté was based on a commercial product manufactured by Mills DA, Oslo, Norway. The main ingredients were farmed Atlantic salmon (Salmo salar), rapeseed oil, water and fish oil (refined cod-liver oil; Denomega Nutritional Oils, Sarpsborg, Norway). Fish oil was added to increase the contents of DHA and EPA to about twice the natural content. The product was gently processed under carefully controlled conditions, high-temperature pasteurised and packed in $\mathrm{Al}$ tubes. Fruit juice was based on concentrates of fruits and berries, with no sugar added. It was developed and delivered by Mills DA, in collaboration with Skånemejerier, Malmø,
Sweden, where it was produced by adding fish oil (refined cod-liver oil; Denomega Nutritional Oils) through a patented technique (patent no. Norge 325446, 'Lipid composition and use thereof'). Fish oil capsules (Peter Møllers Dobbel, Axellus, Norway) were a commercial food supplement of concentrated fish oil (TAG). The capsules were made of gelatine softened with glycerol. All intervention products were obtained from large-scale production and subjected to regular in-house quality tests, including routine sensory analysis and analysis of peroxide value. Sensory analysis was performed by a panel consisting of company employees specially trained to detect oxidative attributes such as rancidity. The participants were told to keep the food products refrigerated in order to ensure oxidative stability throughout the shelf-life period. Fish oil capsules were stored at room temperature. The fatty acid composition of the study products is presented in Table 1. Fatty acid content was analysed by The Norwegian Institute for Food and Environmental Analysis Inc. (Oslo, Norway) using a modified Caviezel method ${ }^{(37)}$. Lipids were extracted using a modified Bligh \& Dyer method ${ }^{(38)}$. Fatty acid concentrations were determined by GLC of fatty acid methyl esters on a fused-silica wall-coated capillary column (Chrompack CP-Wax-52 CB, $25 \mathrm{~mm} \times 0.25 \mathrm{~mm}$ ) in a PerkinElmer Auto System XL (Perkin Elmer Inc., Waltham, MA, USA).

\section{Blood sampling}

Blood from venepuncture was collected after an overnight fast $(\geq 12 \mathrm{~h}$ ). Subjects were instructed to refrain from alcohol consumption and from vigorous physical activity the day before the blood sampling. Serum was obtained from silica gel tubes (BD Vacutainer, Plymouth, Devon, UK), kept at room temperature for at least $30 \mathrm{~min}$ until centrifugation at $1300 \mathrm{~g}$ for $12 \mathrm{~min}$. Plasma was obtained from EDTA tubes (BD Vacutainer) kept on ice and centrifuged at $1300 \mathrm{~g}$ for $10 \mathrm{~min}$ at $10^{\circ} \mathrm{C}$ within $2 \mathrm{~h}$. Samples were kept frozen $\left(-80^{\circ} \mathrm{C}\right)$ until analysis. 


\section{Serum lipids and apolipoproteins}

Analyses of serum total-, LDL- and HDL-cholesterol, TAG, apoA1, and apoB were performed at the routine laboratory at the Department of Medical Biochemistry at Oslo University Hospital, Rikshospitalet, Oslo, Norway (ISO 17025 accredited), using standard methods (Modular System; Roche, Mannheim, Germany).

\section{Plasma fatty acid composition and vitamin $E$ ( $\alpha$-tocopherol)}

Plasma fatty acid composition was analysed by Jurilab Limited, Kuopio, Finland, which used a slight modification of the method described by Nyyssonen et al. ${ }^{(39)}$. Plasma $(250 \mu \mathrm{l})$ fatty acids and $25 \mu \mathrm{l}$ of an internal standard (eicosane $1 \mathrm{mg} / \mathrm{ml}$ in isopropanol) were extracted with $6 \mathrm{ml}$ of methanolchloroform (1:2) and $1.5 \mathrm{ml}$ of water were added. The two phases were separated by centrifugation and the upper phase was discarded. To the chloroform phase, $1 \mathrm{ml}$ of methanol-water (1:1) was added, and this extraction was repeated twice. The chloroform phase was evaporated under $\mathrm{N}_{2}$. For methylation, the remainder was treated with $1.5 \mathrm{ml}$ of sulphuric acid-methanol $(1: 50)$ at $85^{\circ} \mathrm{C}$ for $2 \mathrm{~h}$. The mixture was diluted with $1.5 \mathrm{ml}$ of water and extracted with light petroleum diethyl ether. The fatty acids from the diethyl ether phase were determined using a 6890 GC with an HP-5MS capillary column and interfaced with a 5973 mass selective detector with electron impact ionisation (Agilent Technologies, Palo Alto, CA, USA). For retention time and quantitative standardisation, fatty acids purchased from Nu-Chek-Prep (Elysian, MN, USA) were used.

Plasma $\alpha$-tocopherol was analysed by Vitas AS, Oslo, Norway. Briefly, $100 \mu \mathrm{l}$ of human plasma were diluted with $300 \mu \mathrm{l} 2$-propanol containing the internal standard tocol and butylated hydroxytoluene (BHT) as an antioxidant. After thorough mixing $(15 \mathrm{~min}$ ) and centrifugation $(10 \mathrm{~min}, 4000 \mathrm{~g}$ at $10^{\circ} \mathrm{C}$ ), an aliquot of $1 \mu \mathrm{l}$ was injected from the supernatant into the HPLC system. HPLC was performed with an HP 1100 liquid chromatograph (Agilent Technologies) with an HP1100 fluorescence detector, an emission wavelength of $295 \mathrm{~nm}$ and an excitation wavelength of $330 \mathrm{~nm}$. Tocopherol isomers were separated on a $2 \cdot 1 \mathrm{~mm} \times 250 \mathrm{~mm}$ reversed phase column. The column temperature was $40^{\circ} \mathrm{C}$. A two-point calibration curve was made from an analysis of a $3 \%$ albumin solution enriched with a known concentration of tocopherols. Recovery is $>95 \%$, the method is linear from at least 1 to $200 \mu \mathrm{m}$ and the limit of detection is $0.01 \mu \mathrm{m}$.

\section{Circulating inflammatory markers}

Analysis of serum high-sensitivity C-reactive protein was performed at the routine laboratory at the Department of Medical Biochemistry at Rikshospitalet (ISO17025 accredited), using a standard method (Modular System; Roche). Plasma IL-6, TNF $\alpha$, monocyte chemotactic protein- 1 , interferon- $\gamma$, soluble E-selectin and P-selectin, soluble intracellular adhesion molecule-1 and vascular cell adhesion molecule-1 were determined by Fluorokine ${ }^{\circledR}$ MAP kits from R\&D Systems, Inc. (Minneapolis, MN, USA). Plasma leukotriene $\mathrm{B}_{4}$ was analysed as described previously ${ }^{(29)}$. Due to low sensitivity by the multiplex method, IL- 6 and TNF $\alpha$ were measured using ELISA (R\&D Systems).

\section{Urinary $F_{2}$ isoprostanes}

Spot morning urine samples were analysed for 8 -iso-PGF $2 \alpha$ by a highly specific and validated RIA as described by Basu $^{(40)}$. The cross-reactivity of the 8 -iso- $\mathrm{PGF}_{2 \alpha}$ antibody with 15-keto-13,14-dihydro-8-iso-PGF $2 \alpha, 8$-iso-PGF $2 \beta, \mathrm{PGF}_{2 \alpha}$, 15-keto-PGF $2 \alpha$, 15-keto-13,14-dihydro-PGF $2 \alpha$, thromboxane B-2, $11 \beta-\mathrm{PGF}_{2 \alpha}, 9 \beta-\mathrm{PGF}_{2 \alpha}$ and 8-iso-PGF $3 \alpha$, respectively, was $1 \cdot 7,9 \cdot 8,1 \cdot 1,0 \cdot 01,0 \cdot 01,0 \cdot 1,0 \cdot 03,1 \cdot 8$ and $0 \cdot 6 \%$. The detection limit of the assay was $23 \mathrm{pmol} / \mathrm{l}$. Glomerular filtration rate was assessed as equal to the clearance of creatinine and the urinary levels of 8 -iso- $\mathrm{PGF}_{2 \alpha}$ were adjusted according to creatinine concentration

\section{Statistical methods}

Statistical analyses were performed by LINK Medical Research AS (Oslo, Norway), an independent clinical research organisation contracted for partaking in the conduct of the project. Analyses were performed using SAS ${ }^{\circledR}$ for Windows (version 9.1; SAS Institute Inc., Cary, NC, USA). The nominal level for significance was 5\%. The power calculation was based on observed variations in TAG in a previous parallel study ${ }^{(33)}$. Sample size calculations based on testing a null hypothesis of no difference, using a power of 0.80 and a significance level of $0 \cdot 05$, resulted in the need of including at least forty participants in each group (SAS ${ }^{\circledR}$ Power and Sample Size). This sample size was derived by using an estimated standard deviation of change from baseline of $0.485 \mathrm{mmol} / \mathrm{l}$, and an assumed expected mean change of $0.30 \mathrm{mmol} / 1$ in all groups except the control group that was assumed to have a difference of zero. Due to the potential of participants dropping out of the study, it was decided to include fifty subjects per treatment. The participants were recruited during a period of 1 year. Due to a lower dropout rate than expected, only 179 subjects were recruited. Continuously distributed parameters were analysed by ANOVA. Box plots were checked for deviations between the groups that could severely affect the estimated $P$ values. However, none was found. Whenever the ANOVA test resulted in rejecting the null hypothesis of no difference between the groups, the Scheffé multiple-comparison procedure based on CI was used ${ }^{(41)}$. Within-group changes from baseline to the end of intervention were tested by paired Students $t$ tests. All values are presented as means and standard deviations, unless otherwise stated. In order to detect potential associations between incorporation of DHA and EPA into plasma lipids, the plasma EPA:AA ratio and changes in cardiovascular risk markers, principal component analysis and cross-correlations were performed using Unscrambler ${ }^{\circledR}$ version 9.8 (Camo Inc., Oslo, Norway). 
Table 2. Baseline characteristics and plasma lipids

(Mean values and standard deviations)

\begin{tabular}{|c|c|c|c|c|c|c|c|c|c|}
\hline & \multicolumn{2}{|c|}{ Fish pâté } & \multicolumn{2}{|c|}{ Fruit juice } & \multicolumn{2}{|c|}{ Fish oil capsules } & \multicolumn{2}{|c|}{ Control } & \multirow[b]{2}{*}{$P^{*}$} \\
\hline & Mean & SD & Mean & SD & Mean & SD & Mean & SD & \\
\hline$n$ & \multicolumn{2}{|c|}{44} & \multicolumn{2}{|c|}{38} & \multicolumn{2}{|c|}{40} & \multicolumn{2}{|c|}{37} & \\
\hline Females/males & \multicolumn{2}{|c|}{$27 / 17$} & \multicolumn{2}{|c|}{$28 / 10$} & \multicolumn{2}{|c|}{$31 / 9$} & \multicolumn{2}{|c|}{$25 / 12$} & 0.57 \\
\hline Age (years) & $45 \cdot 0$ & $15 \cdot 1$ & 41.5 & $13 \cdot 6$ & 39.1 & $10 \cdot 9$ & $40 \cdot 3$ & $12 \cdot 7$ & 0.30 \\
\hline $\mathrm{BMI}\left(\mathrm{kg} / \mathrm{m}^{2}\right)$ & $24 \cdot 1$ & 3.0 & 23.9 & $2 \cdot 7$ & 24.5 & $3 \cdot 1$ & $24 \cdot 0$ & $2 \cdot 8$ & 0.86 \\
\hline Systolic BP (mmHg) & 126 & 15 & 121 & 14 & 119 & 12 & 121 & 13 & 0.22 \\
\hline Diastolic BP (mmHg) & 78.4 & $10 \cdot 1$ & $76 \cdot 2$ & $10 \cdot 4$ & $76 \cdot 3$ & $9 \cdot 6$ & $76 \cdot 3$ & $9 \cdot 2$ & 0.71 \\
\hline Total cholesterol $(\mathrm{mmol} / \mathrm{l})$ & $5 \cdot 37$ & 0.79 & 5.03 & 0.86 & 4.93 & 0.78 & 4.95 & 0.93 & 0.18 \\
\hline LDL-cholesterol (mmol/l) & 3.33 & 0.73 & 2.95 & 0.70 & 2.96 & 0.75 & 2.98 & 0.82 & 0.54 \\
\hline HDL-cholesterol (mmol/l) & 1.57 & 0.45 & 1.68 & 0.48 & 1.56 & 0.38 & 1.59 & 0.35 & 0.26 \\
\hline TAG $(\mathrm{mmol} / \mathrm{l})$ & 1.00 & 0.66 & 0.84 & 0.41 & 0.95 & 0.54 & 0.92 & 0.41 & 0.38 \\
\hline
\end{tabular}

$\mathrm{BP}$, blood pressure

* Based on the ANOVA test using means (percentage for sex).

\section{Results}

Overall, 179 subjects were randomised into four groups, and 159 completed the study in accordance with the descriptions given in the protocol. Baseline demographics and clinical characteristics of the study groups are given in Table 2. No differences were observed between the groups. There were no changes in BMI and blood pressure during the intervention period.

\section{Plasma fatty acid profile and serum lipids}

Significant differences in change from baseline between the groups were seen for EPA, docosapentaenoic acid (22:5n-3), DHA and the EPA:AA ratio, demonstrating that the control group had a significantly $(P<0.05)$ smaller change than the three intervention groups (Table 3 ). Also, significant withingroup increases in plasma levels of EPA, docosapentaenoic acid and DHA, and decreased levels of AA were seen in all intervention groups, and the EPA:AA ratio increased markedly (Table 3). EPA increased with 113, 144 and 145\%, respectively, in the fruit juice, fish pâté and fish oil capsule groups. The increases in DHA were somewhat lower, 31, 38 and 50\%, whereas AA decreased with 9, 2 and $7 \%$, respectively, in the fruit juice, fish pâté and fish oil capsule groups. Also, compared with the control group, the increases in EPA, docosapentaenoic acid and DHA were statistically significant, but pairwise comparisons between the three intervention groups gave no significant results. However, the lowest increase in EPA and DHA was observed in the fruit juice group that had the lowest intake (Table 1). There were only minor changes in other plasma fatty acids (Table 3).

The changes seen in the levels of total cholesterol, HDLcholesterol, LDL-cholesterol and TAG were limited (Table 4). There was a trend showing an increase in HDL (5-7\%) in the fruit juice, fish pâté and fish oil capsule groups $(P=0.06-0.07)$. This was in accordance with an observed increase in apoA $(P=0 \cdot 01-0 \cdot 06$; Table 3$)$. Although a few within-group changes were statistically significant, no statistically significant differences in changes between the groups were observed.

\section{Inflammatory markers}

There were no significant changes in the serum inflammatory markers high-sensitivity C-reactive protein, IL-6, TNF $\alpha$, leukotriene $\mathrm{B}_{4}$, monocyte chemotactic protein-1, intracellular adhesion molecule-1, vascular cell adhesion molecule-1, E-selectin and P-selectin (Table 5). A significant withingroup increase was observed for interferon- $\gamma$ in the fruit juice, fish pâté and fish oil capsule groups, but there were no significant differences between the groups (Table 5).

\section{Markers for oxidative stress}

No significant changes in the urine levels of F2-isoprostane between the study groups were observed (Table 6). The plasma level of vitamin E ( $\alpha$-tocopherol) decreased in all groups, including the control group. When calculated in relation to the level of serum TAG, the decrease was statistically significant only in the fish pâté group (Table 6). No significant changes in the plasma levels of $\alpha$-tocopherol were observed between the groups.

\section{Principal component analysis and cross-correlation}

Principal component analysis and cross-correlations revealed no convincing associations between incorporation of DHA and EPA into plasma lipids and markers of inflammation and oxidative stress, or between the plasma EPA:AA ratio and markers of inflammation and oxidative stress (data not shown).

\section{Discussion}

The present randomised, parallel designed intervention study showed that intake of $n-3$ LCPUFA in the form of TAG from fortified foods and concentrated fish oil capsules contributed equally to significant increases in plasma concentrations of EPA and DHA. Still, no significant effects were observed in parameters associated with CVD such as serum lipids and markers of inflammation or oxidative stress. 
Table 3. Fatty acid levels in plasma $(\mu \mathrm{mol} / \mathrm{l})$

(Mean values and standard deviations)

\begin{tabular}{|c|c|c|c|c|c|c|c|c|}
\hline & & \multicolumn{3}{|c|}{ Baseline } & \multicolumn{2}{|c|}{ Change } & \multirow[b]{2}{*}{$P^{*}$} & \multirow[b]{2}{*}{$P \dagger$} \\
\hline & & $n$ & Mean & SD & Mean & SD & & \\
\hline \multirow[t]{4}{*}{ Myristic acid (14:0) } & Fruit juice & 38 & $50 \cdot 8$ & $28 \cdot 6$ & $3 \cdot 6$ & $31 \cdot 8$ & 0.49 & \multirow{4}{*}{0.59} \\
\hline & Fish pâté & 44 & $60 \cdot 5$ & 36.9 & $10 \cdot 0$ & $47 \cdot 9$ & 0.17 & \\
\hline & Fish oil & 40 & $67 \cdot 4$ & $57 \cdot 1$ & $2 \cdot 0$ & $44 \cdot 0$ & 0.77 & \\
\hline & Control & 37 & $60 \cdot 5$ & $29 \cdot 3$ & $-2 \cdot 4$ & $35 \cdot 5$ & 0.69 & \\
\hline \multirow[t]{4}{*}{ Palmitic acid (16:0) } & Fruit juice & 38 & $1667 \cdot 4$ & $467 \cdot 5$ & $-68 \cdot 0$ & $288 \cdot 3$ & $0 \cdot 15$ & \multirow{4}{*}{0.29} \\
\hline & Fish pâté & 44 & 1682.5 & 461.9 & $7 \cdot 2$ & $344 \cdot 2$ & 0.89 & \\
\hline & Fish oil & 40 & $1661 \cdot 0$ & 496.5 & $-139 \cdot 0$ & 409.5 & 0.04 & \\
\hline & Control & 37 & $1652 \cdot 7$ & $374 \cdot 2$ & -74.6 & $322 \cdot 7$ & 0.18 & \\
\hline \multirow[t]{4}{*}{ Palmitoleic acid (16:1n-7) } & Fruit juice & 38 & 69.8 & 33.6 & $-7 \cdot 2$ & $27 \cdot 8$ & 0.12 & \multirow{4}{*}{0.40} \\
\hline & Fish pâté & 44 & $64 \cdot 7$ & $27 \cdot 1$ & 2.4 & $24 \cdot 3$ & 0.52 & \\
\hline & Fish oil & 40 & $67 \cdot 7$ & 35.4 & $-4 \cdot 7$ & $27 \cdot 9$ & 0.29 & \\
\hline & Control & 37 & $68 \cdot 7$ & $33 \cdot 0$ & $-4 \cdot 8$ & $28 \cdot 6$ & 0.31 & \\
\hline \multirow[t]{4}{*}{ Stearic acid $(18: 0)$} & Fruit juice & 38 & $594 \cdot 2$ & $143 \cdot 1$ & $5 \cdot 2$ & $92 \cdot 0$ & 0.73 & \multirow{4}{*}{$0 \cdot 15$} \\
\hline & Fish pâté & 44 & 623.5 & $147 \cdot 4$ & 31.9 & $141 \cdot 6$ & 0.14 & \\
\hline & Fish oil & 40 & $580 \cdot 6$ & $136 \cdot 6$ & $-2 \cdot 2$ & $133 \cdot 2$ & 0.92 & \\
\hline & Control & 37 & $594 \cdot 8$ & $103 \cdot 5$ & -32.4 & $125 \cdot 4$ & 0.12 & \\
\hline \multirow[t]{4}{*}{ Oleic acid $(18: 1 n-9)$} & Fruit juice & 38 & $577 \cdot 6$ & $178 \cdot 2$ & $-48 \cdot 2$ & $130 \cdot 4$ & 0.03 & \multirow{4}{*}{0.58} \\
\hline & Fish pâté & 44 & $585 \cdot 8$ & $177 \cdot 3$ & -2.5 & $208 \cdot 3$ & 0.94 & \\
\hline & Fish oil & 40 & 558.9 & $166 \cdot 1$ & $-42 \cdot 7$ & $154 \cdot 0$ & 0.09 & \\
\hline & Control & 37 & 570.2 & $146 \cdot 8$ & $-23 \cdot 0$ & $151 \cdot 9$ & 0.36 & \\
\hline \multirow[t]{4}{*}{ Linoleic acid (18:2n-6) } & Fruit juice & 38 & 873.9 & $373 \cdot 1$ & -72.5 & $193 \cdot 5$ & 0.03 & \multirow{4}{*}{0.68} \\
\hline & Fish pâté & 44 & 874.0 & 331.8 & $-27 \cdot 6$ & $192 \cdot 0$ & 0.35 & \\
\hline & Fish oil & 40 & $829 \cdot 3$ & $349 \cdot 8$ & $-49 \cdot 6$ & $251 \cdot 3$ & 0.22 & \\
\hline & Control & 37 & $812 \cdot 0$ & $219 \cdot 6$ & $-76 \cdot 3$ & $167 \cdot 5$ & 0.01 & \\
\hline \multirow[t]{4}{*}{$\alpha$-Linolenic acid (18:3n-3) } & Fruit juice & 38 & $62 \cdot 6$ & $20 \cdot 4$ & -6.6 & $19 \cdot 6$ & 0.05 & \multirow{4}{*}{$0 \cdot 12$} \\
\hline & Fish pâté & 44 & $65 \cdot 2$ & 20.5 & 3.0 & $22 \cdot 1$ & 0.37 & \\
\hline & Fish oil & 40 & $61 \cdot 7$ & $17 \cdot 9$ & 0.2 & $20 \cdot 9$ & 0.95 & \\
\hline & Control & 37 & $68 \cdot 3$ & $20 \cdot 4$ & -5.5 & 19.5 & 0.09 & \\
\hline \multirow[t]{4}{*}{ Arachidonic acid $(20: 4 n-6)$} & Fruit juice & 38 & $200 \cdot 1$ & $43 \cdot 2$ & $-18 \cdot 2$ & 35.5 & 0.003 & \multirow{4}{*}{0.17} \\
\hline & Fish pâté & 44 & $197 \cdot 7$ & $44 \cdot 1$ & -3.7 & 31.4 & 0.44 & \\
\hline & Fish oil & 40 & $192 \cdot 6$ & $50 \cdot 0$ & $-14 \cdot 1$ & $29 \cdot 6$ & 0.005 & \\
\hline & Control & 37 & $189 \cdot 8$ & $44 \cdot 2$ & -7.0 & $32 \cdot 3$ & 0.20 & \\
\hline EPA $(20: 5 n-3)$ & Fruit juice & 38 & $30 \cdot 3$ & $19 \cdot 9$ & $34 \cdot 2$ & $29 \cdot 6$ & $<0.0001$ & \\
\hline & Fish pâté & 44 & 35.5 & 20.5 & $51 \cdot 2$ & 31.5 & $<0.0001$ & \\
\hline & Fish oil & 40 & $31 \cdot 2$ & $23 \cdot 1$ & $45 \cdot 2$ & $29 \cdot 6$ & $<0.0001$ & $<0.0001 \ddagger$ \\
\hline & Control & 37 & $43 \cdot 9$ & $40 \cdot 7$ & $-6 \cdot 6$ & $28 \cdot 6$ & 0.17 & \\
\hline Docosapentaenoic acid (22:5n-3) & Fruit juice & 38 & $7 \cdot 9$ & 3.0 & 2.7 & 3.3 & $<0.0001$ & \\
\hline & Fish pâté & 44 & $9 \cdot 1$ & 3.5 & $4 \cdot 1$ & $4 \cdot 3$ & $<0.0001$ & \\
\hline & Fish oil & 40 & $8 \cdot 8$ & $4 \cdot 0$ & 3.9 & $3 \cdot 2$ & 0.0001 & $<0.0001 \ddagger$ \\
\hline & Control & 37 & $9 \cdot 6$ & $5 \cdot 1$ & -1.0 & 3.6 & 0.09 & \\
\hline DHA (22: 6n-3) & Fruit juice & 38 & $46 \cdot 4$ & $20 \cdot 0$ & $14 \cdot 2$ & $19 \cdot 4$ & $<0.0001$ & \\
\hline & Fish pâté & 44 & $52 \cdot 0$ & $26 \cdot 5$ & $19 \cdot 7$ & $18 \cdot 5$ & $<0.0001$ & \\
\hline & Fish oil & 40 & $47 \cdot 0$ & $25 \cdot 7$ & 23.4 & $16 \cdot 5$ & $<0.0001$ & $<0.0001 \ddagger$ \\
\hline & Control & 37 & 57.4 & $23 \cdot 7$ & $-6 \cdot 1$ & $21 \cdot 3$ & 0.09 & \\
\hline EPA:AA & Fruit juice & 38 & 0.15 & 0.09 & 0.20 & 0.13 & $<0.0001$ & \\
\hline & Fish pâté & 44 & 0.18 & 0.09 & 0.28 & 0.17 & $<0.0001$ & \\
\hline & Fish oil & 40 & $0 \cdot 16$ & 0.09 & 0.27 & 0.17 & $<0.0001$ & $<0.0001 \ddagger$ \\
\hline & Control & 37 & 0.22 & 0.16 & -0.02 & 0.09 & 0.21 & \\
\hline
\end{tabular}

* Test of within-group changes (paired $t$ test).

$\dagger$ Test of the null hypothesis of no difference in change from baseline between the groups (ANOVA).

$\ddagger$ Mean change from baseline was significantly different for control group than for the other three groups $(P<0.05)$.

The increase in plasma EPA varied from 113 to $145 \%$ among the intervention groups, whereas the increase in DHA varied from 31 to $50 \%$. However, differences between the groups were not significant, indicating that fish oil capsules, enriched fruit juice and fish pâté represent equivalent sources of dietary EPA and DHA. The assumption that fish intake is more effective in increasing plasma levels of EPA and DHA than fish oil $^{(28,29)}$ was not confirmed in the present study (Table 3). About $50 \%$ of the intake of EPA and DHA in the fish pâté group originated from fish (salmon) and should therefore contribute to higher levels in plasma. However, the present results suggest that incorporation of EPA and DHA into plasma lipids, measured after 7 weeks of supplementation, is independent of the food matrix. This is in accordance with other studies of fortified foods, including foods added with microencapsulated fish oil ${ }^{(42-48)}$. In the present study, the incorporation of EPA and DHA into plasma lipids was somewhat lower, still not significantly lower, in the fruit juice group than in the fish oil group (Table 3), although intakes were quite similar (Table 1). As compliance was 
Table 4. Serum lipids (mmol/l)

(Mean values and standard deviations)

\begin{tabular}{|c|c|c|c|c|c|c|c|c|}
\hline & & \multicolumn{3}{|c|}{ Baseline } & \multicolumn{2}{|c|}{ Change } & \multirow[b]{2}{*}{$P^{\star}$} & \multirow[b]{2}{*}{$P \dagger$} \\
\hline & & $n$ & Mean & SD & Mean & SD & & \\
\hline \multirow[t]{4}{*}{ Total cholesterol } & Fruit juice & 38 & $5 \cdot 03$ & 0.86 & 0.14 & 0.57 & 0.14 & \multirow{4}{*}{0.78} \\
\hline & Fish pâté & 44 & $5 \cdot 37$ & 0.79 & 0.08 & 0.68 & 0.47 & \\
\hline & Fish oil & 40 & 4.93 & 0.78 & 0.20 & 0.42 & 0.01 & \\
\hline & Control & 37 & 4.95 & 0.93 & 0.12 & 0.52 & 0.18 & \\
\hline \multirow[t]{4}{*}{ HDL-cholesterol } & Fruit juice & 38 & 1.68 & 0.48 & 0.07 & 0.23 & 0.06 & \multirow{4}{*}{0.89} \\
\hline & Fish pâté & 44 & 1.57 & 0.45 & 0.05 & 0.17 & 0.07 & \\
\hline & Fish oil & 40 & 1.56 & 0.38 & 0.05 & 0.16 & 0.06 & \\
\hline & Control & 37 & 1.59 & 0.35 & 0.04 & 0.23 & 0.29 & \\
\hline \multirow[t]{4}{*}{ LDL-cholesterol } & Fruit juice & 38 & 2.95 & 0.70 & 0.03 & 0.32 & 0.58 & \multirow{4}{*}{0.35} \\
\hline & Fish pâté & 44 & $3 \cdot 33$ & 0.73 & -0.03 & 0.46 & 0.72 & \\
\hline & Fish oil & 40 & 2.96 & 0.75 & 0.13 & 0.38 & 0.04 & \\
\hline & Control & 37 & 2.98 & 0.82 & 0.05 & 0.36 & 0.44 & \\
\hline \multirow[t]{4}{*}{ TAG } & Fruit juice & 38 & 0.84 & 0.41 & -0.03 & 0.31 & 0.50 & \multirow{4}{*}{$0 \cdot 81$} \\
\hline & Fish pâté & 44 & 1.00 & 0.66 & 0.05 & 0.45 & 0.46 & \\
\hline & Fish oil & 40 & 0.95 & 0.54 & -0.02 & 0.46 & 0.84 & \\
\hline & Control & 37 & 0.92 & 0.41 & 0.02 & 0.43 & 0.82 & \\
\hline \multirow[t]{4}{*}{ ApoA } & Fruit juice & 38 & $1 \cdot 70$ & 0.30 & 0.07 & 0.16 & 0.01 & \multirow{4}{*}{0.71} \\
\hline & Fish pâté & 43 & 1.64 & 0.26 & 0.04 & 0.15 & 0.06 & \\
\hline & Fish oil & 40 & 1.64 & 0.27 & 0.04 & 0.13 & 0.06 & \\
\hline & Control & 37 & 1.68 & 0.27 & 0.07 & 0.17 & 0.02 & \\
\hline \multirow[t]{4}{*}{ ApoB } & Fruit juice & 38 & 0.79 & 0.19 & -0.03 & 0.12 & 0.15 & \multirow{4}{*}{0.65} \\
\hline & Fish pâté & 43 & 0.90 & 0.21 & -0.04 & 0.14 & 0.09 & \\
\hline & Fish oil & 40 & 0.81 & 0.18 & -0.01 & 0.10 & 0.64 & \\
\hline & Control & 37 & 0.79 & 0.20 & -0.01 & 0.10 & 0.41 & \\
\hline
\end{tabular}

* Test of within-group changes (paired $t$ test).

$\dagger$ Test of the null hypothesis of no difference in change from baseline between the groups (ANOVA).

satisfactory in all groups, we have no explanation for the lower incorporation of EPA and DHA after consumption of fruit juice and further research is needed to confirm whether components in fruit juice influence the uptake of fatty acids. Moreover, it cannot be excluded that small variations in product content could have influenced the results. The present results also show that the increase in plasma EPA is larger than the increase in plasma DHA in all groups, despite similar intakes of EPA and DHA (Table 1). This is in accordance with previous studies ${ }^{(28,29,33,34,45)}$, and may be explained by a more strict biological regulation of DHA levels.

The increase in plasma EPA and DHA observed in the intervention groups seemed to be paralleled by a decrease in AA (Table 3), resulting in a marked increase in the EPA:AA ratio. Elevated blood levels of $n$-3 LCPUFA and the EPA:AA ratio, and, in particular, a high percentage of EPA and DHA in erythrocyte membranes (omega-3 index) have been associated with a reduced risk of sudden cardiac death ${ }^{(1,26,49,50)}$. However, despite a marked increase in plasma $n$-3 LCPUFA and the EPA:AA ratio in the present study, no significant effects were observed in parameters known to be associated with CVD such as serum lipids, markers of inflammation and oxidative stress. Neither did multivariate analyses reveal any strong associations between the EPA:AA ratio and any of the measured parameters. The omega- 3 index was not measured in the present study, but future studies should examine whether $n$-3 LCPUFA-enriched food products providing a daily dose of $1 \mathrm{~g}$ EPA and DHA is sufficient to achieve the recommended omega-3 index associated with a reduced risk of $\mathrm{CVD}^{(1,50)}$.

There were no significant effects on serum lipids with the dose given ( $1 \mathrm{~g} / \mathrm{d}$ EPA and DHA), confirming that higher intakes of $2-4 \mathrm{~g} / \mathrm{d}$ may be needed to induce significant reductions in normolipaemic individuals ${ }^{(25,51,52)}$. The reductions seem to depend on the baseline level and are more frequently observed in hypertriacylglycerolaemic persons and CVD patients ${ }^{(6,51,53)}$, which may explain why no change was observed in the present study, which included only healthy volunteers with normal TAG levels. Some studies have shown an increase in HDL-cholesterol levels after the intake of $n$-3 LCPUFA $^{(51,54)}$. In the present study, only a weak indication of positive modulation of HDL was observed (Table 4). The lack of the effects on LDL-cholesterol and serum cholesterol is as expected, assuming that n-3 LCPUFA have a minor effect on serum cholesterol ${ }^{(51)}$. The lack of effect of the intervention products on serum cholesterol could also be confirmed using serum cholesterol-predictive equations $^{(55)}$.

The urine levels of F2-isoprostane did not increase significantly in any of the study groups (Table 6), indicating no changes in oxidative stress. The $\alpha$-tocopherol levels, on the other hand, tended to decrease from baseline in all groups, and more in the intervention groups compared with the control group, but no significant between-group variations were found. Some studies have shown that intakes of EPA and DHA may reduce oxidative stress ${ }^{(56)}$, whereas others have indicated no effect ${ }^{(57)}$, or even increased in vivo lipid 
Table 5. Serum high sensitive C-reactive protein (hsCRP) and plasma inflammatory markers (Mean values and standard deviations)

\begin{tabular}{|c|c|c|c|c|c|c|c|c|}
\hline & \multirow[b]{2}{*}{ Study group } & \multicolumn{3}{|c|}{ Baseline } & \multicolumn{2}{|c|}{ Change } & \multirow[b]{2}{*}{$P^{*}$} & \multirow[b]{2}{*}{$P \dagger$} \\
\hline & & $n$ & Mean & SD & Mean & SD & & \\
\hline \multirow[t]{4}{*}{ hsCRP (mg/l) } & Fruit juice & 38 & $1 \cdot 34$ & 1.69 & -0.09 & 1.64 & 0.72 & \multirow{4}{*}{0.76} \\
\hline & Fish pâté & 44 & 1.53 & 1.81 & 0.08 & $2 \cdot 22$ & 0.81 & \\
\hline & Fish oil & 40 & $1 \cdot 28$ & 1.45 & 0.28 & 1.62 & 0.28 & \\
\hline & Control & 37 & 1.79 & $2 \cdot 27$ & -0.18 & $2 \cdot 50$ & 0.67 & \\
\hline \multirow[t]{4}{*}{ IL-6 (pg/ml) } & Fruit juice & 37 & 1.38 & 1.05 & 0.24 & $1 \cdot 38$ & 0.29 & \multirow{4}{*}{0.62} \\
\hline & Fish pâté & 43 & 1.96 & $2 \cdot 40$ & 0.08 & 1.51 & 0.74 & \\
\hline & Fish oil & 34 & 1.35 & 0.98 & 0.23 & $1 \cdot 38$ & 0.34 & \\
\hline & Control & 36 & $1 \cdot 27$ & 1.05 & -0.14 & $1 \cdot 10$ & 0.44 & \\
\hline \multirow[t]{4}{*}{$\mathrm{TNF}_{\alpha}(\mathrm{pg} / \mathrm{ml})$} & Fruit juice & 37 & 1.91 & $1 \cdot 78$ & -0.06 & 0.69 & 0.61 & \multirow{4}{*}{$0 \cdot 25$} \\
\hline & Fish pâté & 44 & $2 \cdot 01$ & $2 \cdot 68$ & 0.30 & 1.07 & 0.08 & \\
\hline & Fish oil & 35 & 2.04 & $3 \cdot 26$ & 0.03 & 1.04 & 0.88 & \\
\hline & Control & 36 & 1.90 & 1.41 & -0.25 & 1.43 & 0.30 & \\
\hline \multirow[t]{4}{*}{ E-selectin (ng/ml) } & Fruit juice & 38 & $32 \cdot 0$ & 11.3 & $2 \cdot 0$ & $9 \cdot 0$ & 0.17 & \multirow{4}{*}{0.97} \\
\hline & Fish pâté & 42 & $34 \cdot 1$ & $13 \cdot 6$ & $2 \cdot 3$ & $5 \cdot 4$ & 0.01 & \\
\hline & Fish oil & 39 & $32 \cdot 8$ & $11 \cdot 8$ & $2 \cdot 1$ & $7 \cdot 8$ & 0.11 & \\
\hline & Control & 37 & $32 \cdot 4$ & $11 \cdot 8$ & 1.5 & $7 \cdot 0$ & 0.22 & \\
\hline \multirow[t]{4}{*}{ ICAM (ng/ml) } & Fruit juice & 38 & $279 \cdot 5$ & $69 \cdot 4$ & -0.5 & $52 \cdot 7$ & 0.96 & \multirow{4}{*}{0.57} \\
\hline & Fish pâté & 42 & $280 \cdot 4$ & $68 \cdot 8$ & $4 \cdot 2$ & 29.7 & 0.37 & \\
\hline & Fish oil & 39 & $299 \cdot 3$ & 78.5 & $4 \cdot 7$ & $36 \cdot 6$ & 0.44 & \\
\hline & Control & 35 & $282 \cdot 3$ & 59.5 & $-6 \cdot 6$ & $32 \cdot 3$ & 0.23 & \\
\hline \multirow[t]{4}{*}{ MCP-1 (pg/ml) } & Fruit juice & 38 & 31.7 & $10 \cdot 6$ & 0.42 & $16 \cdot 0$ & 0.87 & \multirow{4}{*}{0.68} \\
\hline & Fish pâté & 44 & $32 \cdot 2$ & $12 \cdot 3$ & $2 \cdot 14$ & $18 \cdot 8$ & 0.46 & \\
\hline & Fish oil & 40 & 42.9 & $75 \cdot 7$ & 11.0 & $81 \cdot 7$ & 0.40 & \\
\hline & Control & 37 & $32 \cdot 6$ & $12 \cdot 4$ & 1.56 & $16 \cdot 1$ & 0.56 & \\
\hline \multirow[t]{4}{*}{ INF $\gamma(p g / m l)$} & Fruit juice & 38 & $168 \cdot 0$ & $61 \cdot 23$ & $66 \cdot 66$ & $125 \cdot 4$ & $<0.01$ & \multirow{4}{*}{$0 \cdot 16$} \\
\hline & Fish pâté & 44 & $186 \cdot 3$ & 93.43 & 91.01 & $136 \cdot 4$ & $<0.01$ & \\
\hline & Fish oil & 40 & $179 \cdot 2$ & $80 \cdot 32$ & $59 \cdot 27$ & $119 \cdot 8$ & $<0.01$ & \\
\hline & Control & 37 & $182 \cdot 9$ & $132 \cdot 2$ & $26 \cdot 66$ & $124 \cdot 8$ & 0.20 & \\
\hline
\end{tabular}

ICAM, intracellular adhesion molecule; MCP-1, monocyte chemotactic protein-1; INF $\gamma$, interferon- $\gamma$.

* Test of within-group changes (paired $t$ test).

$\dagger$ Test of the null hypothesis of no difference in change from baseline between the groups (ANOVA).

peroxidation and increased oxidative stress ${ }^{(58,59)}$. The conflicting results may be due to differences in experimental design and different products used. Both dosage and balancing with appropriate intakes of antioxidants seem to be of major importance for the outcome.

We have no explanation for the observed increase in interferon- $\gamma$ levels (Table 5). There was no effect on the great majority of the measured inflammatory markers, and this is in accordance with a large body of evidence indicating that healthy subjects are relatively insensitive to immunomodulation with $n$-3 LCPUFA even at intakes that substantially raise their concentrations in phospholipids of immune cells ${ }^{(60-64)}$. A recent study by Micallef et al. ${ }^{(65)}$, however, has indicated that in healthy individuals, plasma $n-3$ fatty acid concentration is inversely related to high-sensitivity C-reactive protein concentration. No such correlation was found in the present study. Moreover, results from the present study are apparently in contrast to an identically designed previous study where intake of the same fish pâté resulted in significant decreases in inflammatory markers (IL-6, TNF $\alpha$, leukotriene $\mathrm{B}_{4}$ and monocyte chemotactic protein-1) in lipopolysaccharide-stimulated whole blood ${ }^{(33)}$. The different results may be due to

Table 6. Markers of oxidative stress

(Mean values and standard deviations)

\begin{tabular}{|c|c|c|c|c|c|c|c|c|}
\hline & \multirow[b]{2}{*}{ Study group } & \multicolumn{3}{|c|}{ Baseline } & \multicolumn{2}{|c|}{ Change } & \multirow[b]{2}{*}{$P^{*}$} & \multirow[b]{2}{*}{$P \dagger$} \\
\hline & & $n$ & Mean & SD & Mean & $\mathrm{SD}$ & & \\
\hline \multirow[t]{4}{*}{ Urine F2-isoprostane ( $\mathrm{mmol} / \mathrm{mmol}$ creatinine) } & Fruit juice & 38 & 0.42 & 0.29 & -0.03 & $0 \cdot 21$ & 0.31 & \multirow{4}{*}{0.43} \\
\hline & Fish pâté & 44 & 0.38 & 0.30 & -0.04 & 0.22 & 0.28 & \\
\hline & Fish oil & 40 & 0.38 & 0.26 & 0.03 & 0.19 & 0.34 & \\
\hline & Control & 37 & 0.43 & 0.25 & -0.02 & 0.19 & 0.56 & \\
\hline \multirow[t]{4}{*}{ Plasma $\alpha$-tocopherol ( $\mu \mathrm{M} / \mathrm{mmol}$ per litre TAG) } & Fruit juice & 38 & 53.4 & $30 \cdot 6$ & -5.56 & 31.7 & 0.29 & \multirow{4}{*}{0.87} \\
\hline & Fish pâté & 44 & $53 \cdot 8$ & $33 \cdot 2$ & -7.92 & $26 \cdot 2$ & 0.05 & \\
\hline & Fish oil & 40 & $49 \cdot 3$ & $30 \cdot 2$ & -7.02 & $28 \cdot 6$ & 0.13 & \\
\hline & Control & 37 & $46 \cdot 4$ & $27 \cdot 0$ & $-2 \cdot 84$ & $29 \cdot 2$ & 0.56 & \\
\hline
\end{tabular}

* Test of within-group changes (paired $t$ test).

$\dagger$ Test of the null hypothesis of no difference in change from baseline between the groups (ANOVA). 
healthy subjects generally having low levels of circulating inflammatory markers, which makes it hard to detect small changes, whereas with lipopolysaccharide stimulation, much higher measurable levels of the markers are obtained. The intervention period in both studies was 7 weeks, which should ensure sufficient incorporation of $n$-3 LCPUFA into the cell membranes ${ }^{(30,34)}$. Incorporation into the membranes of immune cells may influence their function and the way they respond to external signals ${ }^{(13,66)}$. Whether lipopolysaccharide stimulation is relevant with respect to the in vivo situation needs further investigation and future studies should test the hypothesis that exposure to pathological conditions, such as lipopolysaccharide stimulation, is necessary to reveal the immunomodulatory effects of $n-3$ LCPUFA associated with CVD prevention in healthy individuals.

A weakness of the study is that we cannot rule out that group sizes were too small to detect significant effects on markers of inflammation and oxidative stress. Another weakness is that the study was not blinded. The participants received very different products and this was difficult to hide. In order to avoid confounding factors such as effects of seasonal variation and whether participation itself affected the measured parameters, we included a control group that did not consume any marine $n-3$ supplements or changed their fatty fish intake during the study. A nutritionist carefully instructed them. Also, high baseline $n$-3 LCPUFA plasma concentrations could diminish the effects. Regular fatty fish consumers were therefore not included in the study and all participants were told to terminate their intake of $n-3$ LCPUFA supplements 2 months before inclusion ${ }^{(34)}$. Hence, baseline values were somewhat lower in the present study. Still, plasma $n-3$ LCPUFA calculated as a percentage of total fatty acids were similar to other studies and the observed increases in $n-3$ LCPUFA were within the same range as observed by others ${ }^{(28,33,67,68)}$.

In conclusion, the present study shows that concentrated fish oil in capsules, enriched fruit juice and fish pâté represent suitable delivery systems for EPA and DHA, i.e. the efficiency of the incorporation into plasma lipids was independent of the food matrix. Fruit juice and fish pâté were safe, well tolerated and highly palatable, representing feasible alternatives to meet the nutritional recommendations. Fruit juice can be advised for individuals who do not favour fish or fish oil capsules. The products effectively increase plasma $n$ - 3 LCPUFA content and the EPA:AA ratio. However, significant effects on serum lipids and markers of inflammation and oxidative stress were not observed in this healthy population.

\section{Acknowledgements}

The financial support from the Norwegian companies Aker BioMarine Antarctic AS, Oslo, Norway, Mills DA and Denomega Nutritional Oils AS and the Swedish company Skånemejerier, is highly appreciated. Fish pâté was carefully prepared and made available from Mills DA. Fruit juice was produced by Skånemejerier, and made available from Mills DA. Fish oil added to the products was delivered by Denomega Nutritional Oils. The authors also want to thank Ingunn Hagen
Westgaard and Minna Nurminiemi, Link Medical Research AS, for their contribution to the interpretation and statistical analyses of the data, Professor Bjarne Østerud, Faculty of Health Sciences, University of Troms $\varnothing$, Troms $\varnothing$, Norway, for his contribution to the interpretation and analyses of plasma inflammatory markers, and Professor Jan I. Pedersen, Department of Nutrition, University of Oslo, Oslo, Norway, for valuable discussions and reading the manuscript. We also want to thank the volunteers who participated in the study. Conflict of interest: some of the authors are employed by the companies that delivered products to the trial. A. L. and E. F. are employees at Mills DA, Lena Nyberg at Skånemejerier, Hogne Vik and N. H. at Aker BioMarine Antarctic AS and E. O. at Denomega Nutritional Oils AS. B. K. worked for Mills DA when the project started. B. K. contributed to the design of the experiment, analysis and interpretation of the data and writing of the manuscript. A. L. contributed to the design of the experiment, product development, analysis and interpretation of the data and revising of the manuscript. K.-E. E. contributed to the design of the experiment, interpretation of the data and writing of the manuscript. E. F. contributed to the collection of the data, analysis and interpretation of the data and revising of the manuscript. T. H. contributed to the planning of the study design, statistical analyses of the data and revising of the manuscript. $H$. V. contributed to the planning of the study design, discussion and analysis of the study results, interpretation of the data, and writing of the manuscript. N. H. contributed to the initial planning of the study design and statistical analytical plan, interpretation of the data and revision of the manuscript. T.-A. H. contributed to the initial planning of the project, interpretation of some of the results and writing of the manuscript. S. B. contributed to the analysis and interpretation of the data and revising of the manuscript. E. O. contributed to the interpretation of the data and revising of the manuscript. I. S. contributed to consultation, discussion of the results and writing of the manuscript. L. N. contributed to the design of the experiment, interpretation of the data and revising of the manuscript. E. E. contributed to the conduction of the trial, collection of the data and writing of the manuscript. S. M. U. contributed to the conduction of the trial, collection of the data, analysis and interpretation of the data and writing of the manuscript.

\section{References}

1. Harris WS (2008) The omega-3 index as a risk factor for coronary heart disease. Am J Clin Nutr 87, 1997s-2002s.

2. Mozaffarian D (2008) Fish and $n$-3 fatty acids for the prevention of fatal coronary heart disease and sudden cardiac death. Am J Clin Nutr 87, 1991s-1996s.

3. Calder PC (2006) n-3 Polyunsaturated fatty acids, inflammation, and inflammatory diseases. Am J Clin Nutr $\mathbf{8 3}$ 1505s-1519s.

4. Cunnane SC, Plourde M, Pifferi F, et al. (2009) Fish, docosahexaenoic acid and Alzheimer's disease. Prog Lipid Res $\mathbf{4 8}$, 239-256.

5. Appleton KM, Rogers PJ \& Ness AR (2010) Updated systematic review and meta-analysis of the effects of $n-3$ long-chain

tional Oils. The authors also want to thank Ingunn Hagen 
polyunsaturated fatty acids on depressed mood. Am J Clin Nutr 91, 757-770.

6. Valagussa F, Franzosi MG, Geraci E, et al. (1999) Dietary supplementation with $n-3$ polyunsaturated fatty acids and vitamin $\mathrm{E}$ after myocardial infarction: results of the GISSIPrevenzione trial. Lancet 354, 447-455.

7. Lemaitre RN, King IB, Mozaffarian D, et al. (2003) n-3 Polyunsaturated fatty acids, fatal ischemic heart disease, and nonfatal myocardial infarction in older adults: the Cardiovascular Health Study. Am J Clin Nutr 77, 319-325.

8. Mozaffarian D (2007) Fish oil and prevention of atrial fibrillation. J Am Coll Cardiol 50, 1513-1514.

9. Cicero AFG, Ertek S \& Borghi C (2009) Omega-3 polyunsaturated fatty acids: their potential role in blood pressure prevention and management. Curr Vasc Pharmacol 7, 330-337.

10. Thies F, Nebe-von-Caron G, Powell JR, et al. (2001) Dietary supplementation with eicosapentaenoic acid, but not with other long-chain $n$-3 or $n-6$ polyunsaturated fatty acids, decreases natural killer cell activity in healthy subjects aged > 55y. Am J Clin Nutr 73, 539-548.

11. Zampelas A, Panagiotakos DB, Pitsavos C, et al. (2005) Fish consumption among healthy adults is associated with decreased levels of inflammatory markers related to cardiovascular disease - The ATTICA study. J Am Coll Cardiol 46, 120-124.

12. Paulo MC, Andrade AM, Andrade ML, et al. (2008) Influence of $n-3$ polyunsaturated fatty acids on soluble cellular adhesion molecules as biomarkers of cardiovascular risk in young healthy subjects. Nutr Metab Cardiovasc Dis 18, 664-670.

13. Calder PC (2008) The relationship between the fatty acid composition of immune cells and their function. Prostaglandins Leukot Essent Fatty Acids 79, 101-108.

14. Clifton P (2009) Dietary fatty acids and inflammation. Nutr Dietetics 66, 7-11.

15. Damsgaard CT, Lauritzen L, Calder PC, et al. (2009) Reduced ex vivo interleukin-6 production by dietary fish oil is not modified by linoleic acid intake in healthy men. $J$ Nutr 139, 1410-1414.

16. Troseid M, Arnesen H, Hjerkinn EM, et al. (2009) Serum levels of interleukin-18 are reduced by diet and $n-3$ fatty acid intervention in elderly high-risk men. Metabolism $\mathbf{5 8}$, 1543-1549.

17. Myhrstad MC, Retterstol K, Telle-Hansen VH, et al. (2011) Effect of marine $n-3$ fatty acids on circulating inflammatory markers in healthy subjects and subjects with cardiovascular risk factors. Inflamm Res 60, 309-319.

18. Suzukawa M, Abbey M, Howe PRC, et al. (1995) Effects of fish-oil fatty-acids on low-density-lipoprotein size, oxidizability, and uptake by macrophages. J Lipid Res 36, 473-484

19. Puiggros C, Chacon P, Armadans LI, et al. (2002) Effects of oleic-rich and omega-3-rich diets on serum lipid pattern and lipid oxidation in mildly hypercholesterolemic patients. Clin Nutr 21, 79-87.

20. Turner R, McLean CH \& Silvers KM (2006) Are the health benefits of fish oils limited by products of oxidation? Nutr Res Rev 19, 53-62.

21. Basu S (2004) Isoprostanes: novel bioactive products of lipid peroxidation. Free Radic Res 38, 105-122.

22. Basu S (2008) F2-isoprostanes in human health and diseases: from molecular mechanisms to clinical implications. Antioxid Redox Signal 10, 1405-1434.

23. Daryani A, Basu S, Becker W, et al. (2007) Antioxidant intake, oxidative stress and inflammation among immigrant women from the Middle East living in Sweden: associations with cardiovascular risk factors. Nutr Metab Cardiovasc Dis 17, 748-756.

24. Kim JY, Hyun YJ, Jang Y, et al. (2008) Lipoprotein-associated phospholipase A2 activity is associated with coronary artery disease and markers of oxidative stress: a case-control study. Am J Clin Nutr 88, 630-637.

25. Kris-Etherton PM \& Hill AM (2008) n-3 Fatty acids: food or supplements? J Am Diet Assoc 108, 1125-1130.

26. Rupp H, Wagner D, Rupp T, et al. (2004) Risk stratification by the "EPA+DHA level" and the "EPA/AA ratio" focus on anti-inflammatory and antiarrhythmogenic effects of longchain omega-3 fatty acids. Herz 29, 673-685.

27. EFSA (2009) Labelling reference intake values for $n-3$ and n-6 polyunsaturated fatty acids. EFSA J 1176, 1-11.

28. Visioli F, Rise P, Barassi MC, et al. (2003) Dietary intake of fish vs. formulations leads to higher plasma concentrations of $n$-3 fatty acids. Lipids 38, 415-418.

29. Elvevoll EO, Barstad H, Breimo ES, et al. (2006) Enhanced incorporation of $n-3$ fatty acids from fish compared with fish oils. Lipids 41, 1109-1114.

30. Harris WS, Pottala JV, Sands SA, et al. (2007) Comparison of the effects of fish and fish-oil capsules on the $n 3$ fatty acid content of blood cells and plasma phospholipids. Am J Clin Nutr 86, 1621-1625.

31. Schram LB, Nielsen CJ, Porsgaard T, et al. (2007) Food matrices affect the bioavailability of ( $n-3)$ polyunsaturated fatty acids in a single meal study in humans. Food Res Int 40, 1062-1068.

32. Mun S, Decker EA \& McClements DJ (2007) Influence of emulsifier type on in vitro digestibility of lipid droplets by pancreatic lipase. Food Res Int 40, 770-781.

33. Elvevoll EO, Eilertsen KE, Brox J, et al. (2008) Seafood diets: hypolipidemic and antiatherogenic effects of taurine and $n-3$ fatty acids. Atherosclerosis 200, 396-402.

34. Katan MB, Deslypere JP, vanBirgelen APJM, et al. (1997) Kinetics of the incorporation of dietary fatty acids into serum cholesteryl esters, erythrocyte membranes, and adipose tissue: an 18-month controlled study. J Lipid Res 38, 2012-2022.

35. Andersen LF, Nes M, Lillegaard IT, et al. (1995) Evaluation of a quantitative food frequency questionnaire used in a group of Norwegian adolescents. Eur J Clin Nutr 49 , $543-554$.

36. Ulven SM, Kirkhus B, Lamglait A, et al. (2011) Metabolic effects of krill oil are essentially similar to those of fish oil but at lower dose of EPA and DHA, in healthy volunteers. Lipids 46, 37-46.

37. Pendl R, Bauer M, Caviezel R, et al. (1998) Determination of total fat in foods and feeds by the caviezel method, based on a gas chromatographic technique. J AOAC Int $\mathbf{8 1}$, 907-917.

38. Bligh EG \& Dyer WJ (1959) A rapid method of total lipid extraction and purification. Can J Biochem Physiol 37, 911-917.

39. Nyyssonen K, Kaikkonen J \& Salonen JT (1996) Characterization and determinants of an electronegatively charged lowdensity lipoprotein in human plasma. Scand J Clin Lab Invest 56, 681-689.

40. Basu S (1998) Radioimmunoassay of 8-iso-prostaglandin F-2 alpha: an index for oxidative injury via free radical catalysed lipid peroxidation. Prostaglandins Leukot Essent Fatty Acids 58, 319-325.

41. SAS Institute Inc. (2010) SAS/STAT 9.22 User's Guide, pp. 3074. Cary, NC: SAS Institute Inc.

42. Mantzioris E, Cleland LG, Gibson RA, et al. (2000) Biochemical effects of a diet containing foods enriched with $n$ - 3 fatty acids. Am J Clin Nutr 72, 42-48. 
43. Wallace JMW, McCabe AT, Robson PJ, et al. (2000) Bioavailability of $n-3$ polyunsaturated fatty acids (PUFA) in foods enriched with microencapsulated fish oil. Ann Nutr Metab 44, 157-162.

44. Yep YL, Li D, Mann NJ, et al. (2002) Bread enriched with microencapsulated tuna oil increases plasma docosahexaenoic acid and total omega-3 fatty acids in humans. Asia Pac J Clin Nutr 11, 285-291.

45. Metcalf RG, James MJ, Mantzioris E, et al. (2003) A practical approach to increasing intakes of $n-3$ polyunsaturated fatty acids: use of novel foods enriched with $n-3$ fats. Eur J Clin Nutr 57, 1605-1612.

46. Higgins S, Carroll YL, O'Brien NM, et al. (1999) Use of microencapsulated fish oil as a means of increasing $n-3$ polyunsaturated fatty acid intake. J Hum Nutr Dietetics 12, 265-271.

47. Arterburn LM, Oken HA, Hoffman JP, et al. (2007) Bioequivalence of docosahexaenoic acid from different algal oils in capsules and in a DHA-fortified food. Lipids 42, 1011-1024.

48. Earnest CP, Hammar MK, Munsey M, et al. (2009) Microencapsulated foods as a functional delivery vehicle for omega-3 fatty acids: a pilot study. J Int Soc Sports Nutr 6, 12.

49. Albert CM, Campos H, Stampfer MJ, et al. (2002) Blood levels of long-chain $n-3$ fatty acids and the risk of sudden death. $N$ Engl J Med 346, 1113-1118.

50. von Schacky C \& Harris WS (2007) Cardiovascular risk and the omega-3 index. J Cardiovasc Med (Hagerstown) 8, Suppl. 1, S46-S49.

51. Harris WS (1997) $n-3$ Fatty acids and serum lipoproteins: human studies. Am J Clin Nutr 65, S1645-S1654.

52. Kris-Etherton PM, Harris WS, Appel LJ, et al. (2002) Fish consumption, fish oil, omega-3 fatty acids, and cardiovascular disease. Circulation 106, 2747-2757.

53. Seierstad SL, Seljeflot I, Johansen O, et al. (2005) Dietary intake of differently fed salmon; the influence on markers of human atherosclerosis. Eur J Clin Invest 35, 52-59.

54. Balk EM, Lichtenstein AH, Chung M, et al. (2006) Effects of omega-3 fatty acids on serum markers of cardiovascular disease risk: a systematic review. Atherosclerosis 189, 19-30.

55. Muller H, Kirkhus B \& Pedersen JI (2001) Serum cholesterol predictive equations with special emphasis on trans and saturated fatty acids: an analysis from designed controlled studies. Lipids 36, 783-791.
56. von Schacky C (2007) n-3 PUFA in CVD: influence of cytokine polymorphism. Proc Nutr Soc 66, 166-170.

57. Tholstrup T, Hellgren LI, Petersen M, et al. (2004) A solid dietary fat containing fish oil redistributes lipoprotein subclasses without increasing oxidative stress in men. $J$ Nutr 134, 1051-1057.

58. Stalenhoef AFH, de Graaf J, Wittekoek ME, et al. (2000) The effect of concentrated $n-3$ fatty acids versus gemfibrozil on plasma lipoproteins, low density lipoprotein heterogeneity and oxidizability in patients with hypertrygliceridemia. Atherosclerosis 153, 129-138.

59. Harats D, Dabach Y, Hollander G, et al. (1991) Fish oil ingestion in smokers and nonsmokers enhances peroxidation of plasma-lipoproteins. Atherosclerosis 90, 127-139.

60. Schmidt EB, Arnesen H, de Caterina R, et al. (2005) Marine $n-3$ polyunsaturated fatty acids and coronary heart disease. Part I. Background, epidemiology, animal data, effects on risk factors and safety. Thromb Res 115, 163-170.

61. Sijben JWC \& Calder PC (2007) Differential immunomodulation with long-chain $n$-3 PUFA in health and chronic disease. Proc Nutr Soc 66, 237-259.

62. Fritsche K (2006) Fatty acids as modulators of the immune response. Annu Rev Nutr 26, 45-73.

63. Pot GK, Brouwer IA, Enneman A, et al. (2009) No effect of fish oil supplementation on serum inflammatory markers and their interrelationships: a randomized controlled trial in healthy, middle-aged individuals. Eur J Clin Nutr 63, 1353-1359.

64. Hallund J, Madsen BO, Bugel SH, et al. (2010) The effect of farmed trout on cardiovascular risk markers in healthy men. Br J Nutr 104, 1528-1536.

65. Micallef MA, Munro IA \& Garg ML (2009) An inverse relationship between plasma $n-3$ fatty acids and C-reactive protein in healthy individuals. Eur J Clin Nutr 63, 1154-1156.

66. Yaqoob P (2010) Mechanisms underlying the immunomodulatory effects of n-3 PUFA. Proc Nutr Soc 69, 311-315.

67. Zhang J, Wang C, Li L, et al. (2010) Inclusion of Atlantic salmon in the Chinese diet reduces cardiovascular disease risk markers in dyslipidemic adult men. Nutr Res 30, 447-454.

68. Rosell MS, Lloyd-Wright Z, Appleby PN, et al. (2005) Longchain $n$-3 polyunsaturated fatty acids in plasma in British meat-eating, vegetarian, and vegan men. Am J Clin Nutr 82, 327-334. 